\title{
Propofol protects hippocampal neurons from apoptosis in ischemic brain injury by increasing GLT-1 expression and inhibiting the activation of NMDAR via the JNK/Akt signaling pathway
}

\author{
HONG-YAN GONG ${ }^{1}$, FANG ZHENG ${ }^{2}$, CHAO ZHANG $^{3}$, XI-YAN CHEN $^{4}$, JING-JING LIU $^{1}$ and XIU-QIN YUE ${ }^{1}$ \\ Departments of ${ }^{1}$ Anesthesia, ${ }^{2}$ Ultrasound, ${ }^{3}$ Orthopaedics and ${ }^{4}$ Emergency, \\ The First Affiliated Hospital of Xinxiang Medical University, Weihui, Henan 453100, P.R. China
}

Received December 19, 2015; Accepted June 21, 2016

DOI: $10.3892 / \mathrm{ijmm} .2016 .2663$

\begin{abstract}
Ischemic brain injury (IBI) can cause nerve injury and is a leading cause of morbidity and mortality worldwide. The neuroprotective effects of propofol against IBI have been previously demonstrated. However, the neuroprotective effects of propofol on hippocampal neurons are not yet entirely clear. In the present study, models of IBI were established in hypoxiaexposed hippocampal neuronal cells. Cell viability assay and apoptosis assay were performed to examine the neuroprotective effects of propofol on hippocampal neurons in IBI. A significant decrease in cell viability and a significant increase in cell apoptosis were observed in the IBI group compared with the control group, accompanied by a decrease in glial glutamate transporter-1 (GLT-1) expression as determined by RT-qPCR and western blot analysis. The effects of IBI were reversed by propofol treatment. The siRNA-mediated knockdown of GLT-1 in the hypoxia-exposed hippocampal neuronal cells led to an increase in cell apoptosis, Jun N-terminal kinase (JNK) activation and N-methyl-D-aspartate (NMDA) receptor (NR1 and NR2B) activation, as well as to a decrease in cell viability and a decrease in Akt activation. The effects of RNA interference-mediated GLT-1 gene silencing on cell viability, JNK activation, NMDAR activation, cell apoptosis and Akt activation in the hippocampal neuronal cells were slightly reversed by propofol treatment. The JNK agonist, anisomycin, and the Akt inhibitor, LY294002, both significantly blocked the effects of propofol on hippocampal neuronal cell viability and apoptosis in IBI. The decrease in JNK activation and the increase in Akt activation caused by GLT-1 overexpression were reversed by NMDA. Collectively, our findings suggest that propofol treatment protects hippocampal neurons against IBI by enhancing
\end{abstract}

Correspondence to: Dr Xiu-Qin Yue, Department of Anesthesia, The First Affiliated Hospital of Xinxiang Medical University, 88 Jiankang Road, Weihui, Henan 453100, P.R. China

E-mail: xiuqinyueok@163.com

Key words: ischemic brain injury, propofol, glial glutamate transporter-1, Jun N-terminal kinase, Akt
GLT-1 expression and inhibiting the activation of NMDAR via the JNK/Akt signaling pathway.

\section{Introduction}

Ischemic brain injury (IBI) is a complex pathology that leads to inflammation (1), apoptosis (2) and excitotoxicity (3) and causes neuronal damage. Excessive extracellular glutamate is released in brain ischemia. As one of the neurotransmitters, high-level glutamate causes excitotoxicity and cell death (4). Glutamate-mediated excitotoxicity is rescued by glutamate transporters (5), such as glial glutamate transporter-1 (GLT-1).

GLT-1 is a transporter that can regulate glutamate homeostasis in the extracellular fluid, which lacks the enzyme to catabolize glutamate (6). GLT-1 is so named due to its main distribution on glial cells. GLT-1 can remove excess glutamate and reduce excitotoxicity (7). Over $90 \%$ of glutamate uptake is carried out by GLT-1 in the adult forebrain (6). GLT-1 knockdown in the hippocampal CA1 region has been shown to lead to high levels of extracellular glutamate (8). A study showed that ischemia-reperfusion led to a significant decrease in GLT-1 in the hippocampal CA1 region (9). The excitotoxicity caused by the decrease in GLT-1 levels can be reversed by cerebral ischemic preconditioning and transient sublethal cerebral ischemia, which protects neurons against brain ischemic damage (10). There is evidence to suggest that the upregulation of GLT-1 results in neuroprotection against excitotoxicity and ischemic injury (11-13). In addition, a high level of GLT-1 reduces the activity of N-methyl-D-aspartate (NMDA) receptors in morphine-induced analgesia in rats (14). The excessive activation of NMDA leading to apoptosis and excitotoxicity underlies normal synaptic plasticity (15). These findings indicate that NMDA-induced excitotoxicity plays a critical role in neuronal damage, such as ischemia-reperfusion insults (16). The effect of NMDA-induced excitotoxicity can be attenuated by the upregulation of GLT-1 (17).

Propofol, as one of the common anesthetics, is widely used in clinical settings. The neuroprotective effects of propofol have previously been demonstrated $(18,19)$. Hypoxia-induced hippocampal neuron injury was also attenuated by propofol (20). A recent study demonstrated that propofol upregulates the expression of GLT-1, which leads to a decrease in the concentration 
of glutamate in the hippocampus of depressed rats (21). In a study on arterioles, propofol reduced both the dilatation and superoxide production caused by NMDA (22). In another study, NMDA-induced excitotoxicity was attenuated by treatment with propofol in cultured rat cortical neurons (23).

However, it remains unclear as to whether propofol protects hippocampal neurons by upregulating GLT-1 expression in IBI. In the present study, we explored the underlying mechanisms and the effect of propofol on hippocampal neurons in IBI.

\section{Materials and methods}

Primary hippocampal neuronal culture. The use of all animals in the experiments conformed to the guidelines of the National Council on Animal Care. All experiments were conducted in accordance with the rules of the Committee on the Ethics of Animal Experimentation of the First Affiliated Hospital of Xinxiang Medical University, Weihui, China. Pregnant Wistar rats $(n=20$; the Laboratory Animal Center of the First Affiliated Hospital of Xinxiang Medical University, Henan, China) were sacrificed by cervical dislocation, and 15-17 day embryos (E15E17) were removed and the brains were rapidly collected, as previously described $(24,25)$. These hippocampal tissues were dissociated in $0.125 \%$ trypsin-EDTA solution for $20 \mathrm{~min}$ at $37^{\circ} \mathrm{C}$ in an incubator. The cells were collected by centrifugation. The cells seeded on 6-well dishes pre-coated with poly-L-lysine and chamber slides were cultured in a $5 \% \mathrm{CO}_{2}$ incubator at $37^{\circ} \mathrm{C}$ at a density of $1 \times 10^{6} / \mathrm{ml}$, and supplied with $1 \%$ glutamine, $10 \%$ heatinactivated fetal bovine serum, $10 \%$ horse serum, $1 \%$ penicillin and streptomycin. After $24 \mathrm{~h}, 100 \mathrm{mM}$ cytosine arabinoside was added to the culture medium to inhibit neuroglial cells. The culture medium supplied with $2 \%$ B27 and 1\% N2, was changed every 3 days. All reagents were purchased from Sigma (St. Louis, MO, USA). Culture cells were used after 7 days in vitro.

Establishment of model of IBI by exposure to hypoxia. After 7 days of normoxic cultivation (20\% oxygen), the culture conditions of the hippocampal neurons were changed to $5 \% \mathrm{CO}_{2}$ and $95 \% \mathrm{~N}_{2}$ at $37^{\circ} \mathrm{C}$ for $3 \mathrm{~h} \mathrm{(26)}$ to establish the model of IBI. After $2 \mathrm{~h}$ of incubation under hypoxic conditions, the hippocampal neuronal cells were treated with propofol (Sigma; $15 \mu \mathrm{M}$ ) and either pre-incubated (or not) with inhibitors of PI3-kinase (LY294002; Sigma; $5 \mu \mathrm{M}$ ) or anisomycin (Sigma; $5 \mu \mathrm{M}$ ) followed by culture for $1 \mathrm{~h}$.

Reverse transcription-quantitative polymerase chain reaction $(R T-q P C R)$. The hippocampal neuronal cells cultured for 7 days were used for the detection of the mRNA levels of GLT-1. The RNeasy mini kit (Qiagen, Hilden, Germany) and PrimeScript $^{\mathrm{TM}}$ RT reagent kit (Takara Biotechnology, Dalian, China) were used for total RNA extraction, reverse transcription and PCR amplification. Gene-specific primers for GLT-1 (forward, 5'-AGTATGTGGCGGGCTGCTTC-3' and reverse, 5'-GGAA ATGATGAGAGGGAGGATGAG-3'); NR 1 (forward, 5'-GCACGCCTTTATCTGGGACTC-3' and reverse, 5'-GTCGGGCTCTGCTCTACCACT-3'); NR2A (forward, 5'-GCTACACACTCTGCACCAATT-3' and reverse, 5'-CACCTGATAGCCTTCCTCAG TGA-3'); NR2B (forward, 5'-TCCGTCTTTCTTATGTGGATATGC-3' and reverse, 5'-CCTCTAGGCGGACAGATTAAGG-3') and the internal control primers for glyceraldehyde 3-phosphate dehydrogenase (GAPDH) (forward, 5'-GCCAAAAGGGTCATCATCT CTG-3' and reverse, 5'-CATGCCAGTGAGCTTCCCGT-3') were synthesized by Sangon Biotech (Sangon, Shanghai, China). The mRNA expression levels were normalized to those of GAPDH and were calculated using the $2^{-\Delta \Delta \mathrm{Ct}}$ method, as previously described (27).

GLT-1 overexpression and silencing. cDNA for rat GLT-1 was amplified by PCR. The obtained GLT-1 cDNA was subcloned into the pIRES vector (Invitrogen Life Technologies, Carlsbad, CA, USA) to generate the overexpression vector of GLT-1, named GLT-1-pIRES. The GLT-1-pIRES vector was transfected into the hippocampal neurons pre-incubated (or not) with NMDA (Sigma; $5 \mu \mathrm{M}$ ) using Lipofectamine 2000 (Invitrogen) following the manufacturer's instructions. The empty pIRES vector was used as a control. Small interfering RNA (siRNA) against GLT-1 and a negative control siRNA were purchased from Santa Cruz Biotechnology, Inc. (Santa Cruz, CA, USA). The siRNA targeting GLT-1 were transfected into the hippocampal neurons using Lipofectamine 2000 according to the manufacturer's instructions. Six hours after transfection, the cells were used for the subsequent experiments.

Cell viability assay. Hippocampal neuronal cell viability was assessed by AlamarBlue (Sigma). The cells were plated onto 96-well plates at a density of $1 \times 10^{6}$ cells/well and $10 \mu \mathrm{l}$ AlamarBlue reagent were added to each well followed by incubatiohn at $5 \% \mathrm{CO}_{2}$ and $37^{\circ} \mathrm{C}$. After $4 \mathrm{~h}$, the percentage reduction in light absorption at 570 and $600 \mathrm{~nm}$ was detected in each well for oxidized and reduced AlamarBlue using a microplate reader (Thermo Fisher Scientific, Waltham, MA, USA). The experiment was performed at least 3 times. Results were calculated as previously described (28).

Cell apoptosis assay. The Annexin V-FITC/propidium iodide (PI) apoptosis detection kit (Sigma) was used to detect cell apoptosis using a flow cytometer (Gibco, Rockville, MD, USA). Briefly, the hippocampal neuron cell medium plated in 6-well dishes was removed and trypsin was added. The cells were then washed twice with phosphate-buffered saline (PBS). Annexin V-FITC and PI were added and the cells were incubated for $30 \mathrm{~min}$ at $37^{\circ} \mathrm{C}$. The stained neurons were analyzed by flow cytometry and the rate of cell apoptosis was determined.

Western blot analysis. Total proteins were extracted from the cultured hippocampal neuronal cells in RIPA lysis buffer (Sigma). The BCA protein assay kit (Beyotime Institute of Biotechnology, Haimen, China) was used to measure the protein concentration using a microplate reader (Thermo Fisher Scientific, Uppsala, Sweden). Protein $(20 \mu \mathrm{g})$ from each sample was separated by $12 \%$ sodium dodecyl sulfate-polyacrylamide gel electrophoresis (SDS-PAGE) gel and transferred to a nitrocellulose membrane. Primary antibodies (Abcam, Cambridge, UK) against GLT-1 (Cat. no. ab178401), Jun N-terminal kinase (JNK; Cat. no. ab179461), p-JNK (Cat. no. ab76572), p38 (Cat. no. ab31828), p-p38 (Cat. no. ab4822), extracellular signalregulated kinase 1/2 (ERK1/2; Cat. no. ab54230), p-ERK1/2 (Cat.no.ab47339), Akt(Cat.no.ab8805),p-Akt(Cat.no.ab38449), NR1 (Cat. no. ab17345), NR2A (Cat. no. ab14596), NR2B 

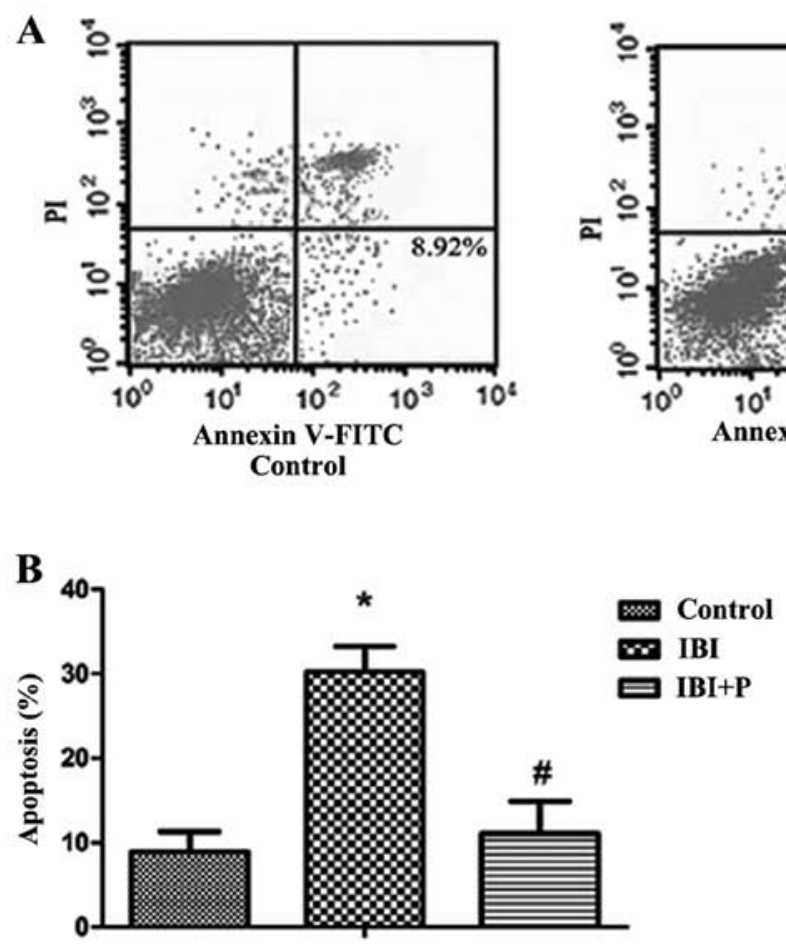
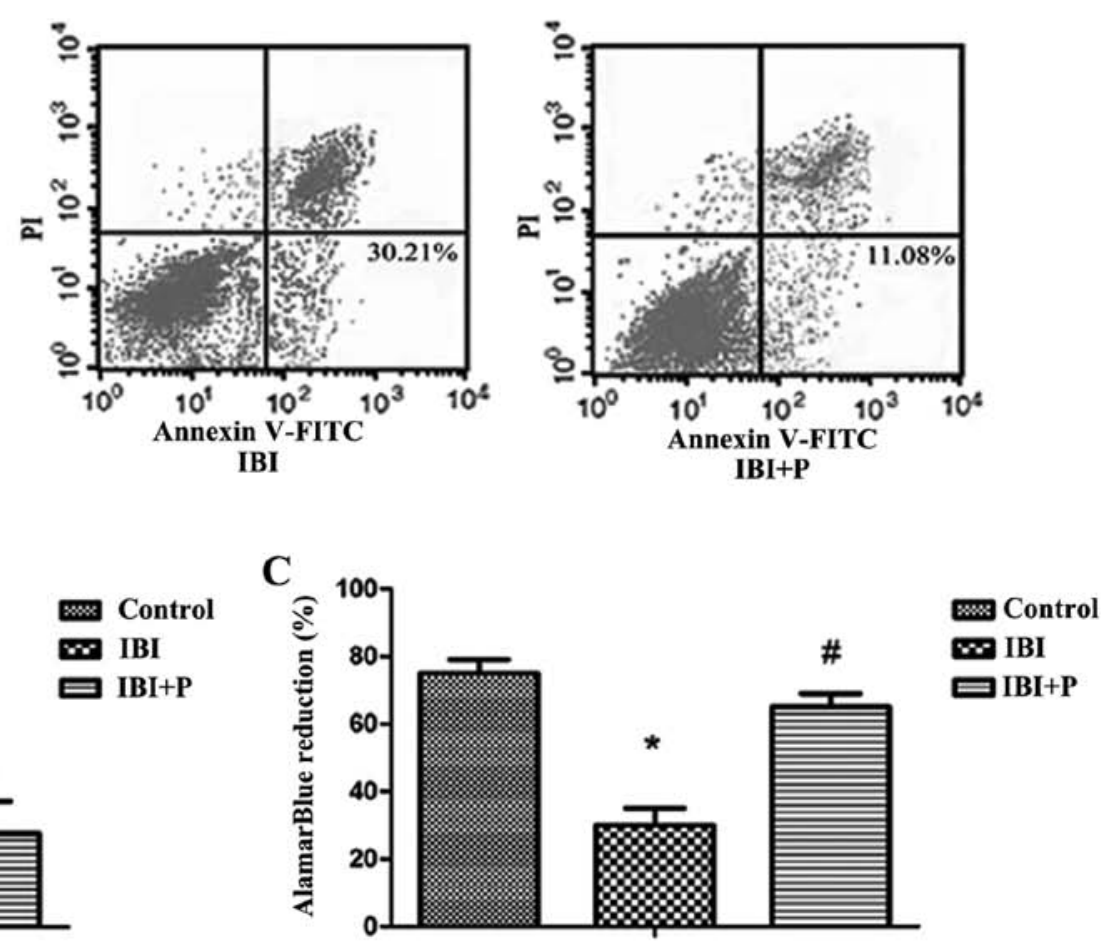

Figure 1. Protective effects of propofol on hippocampal neurons in ischemic brain injury (IBI). A cell model of IBI was established by exposing hippocampal neuronal cells to hypoxia. Cells in the IBI $+\mathrm{P}$ group received $15 \mu \mathrm{M}$ propofol on the basis of IBI. (A) Annexin V-FITC/propidium iodide (PI) was used to detect cell apoptosis as shown in the dual-parameter fluorescent dot plots by flow cytometry. (B) Quantitative graphs of (A). (C) Hippocampal neuron cell viability was assessed by AlamarBlue. ${ }^{*} \mathrm{P}<0.05$ vs. control group; ${ }^{\text {}} \mathrm{P}<0.05$ vs. IBI group.

(Cat. no. ab28373) and $\beta$-actin (Cat. no. ab8226) were used to detect the protein levels. The horseradish peroxidase (HRP)labeled secondary antibodies were purchased from Abcam. $\beta$-actin was utilized as an internal control. The gray value was analyzed using ImageJ software.

Statistical analysis. All results are reported as the means \pm SD. Two-group comparisons were analyzed using the Student's t-test. A value of $\mathrm{P}<0.05$ was considered to indicate a statistically significant difference.

\section{Results}

Neuroprotective effects of propofol on hippocampal neurons. To investigate the effects of propofol on IBI, a model of IBI was established using primary hippocampal neurons. We found that exposure to hypoxia induced a significant decrease in cell viability and an increase in cell apoptosis in the IBI group compared with the control group $(\mathrm{P}<0.05$; Fig. 1). The increase in cell apoptosis and the decrease in cell viability induced by IBI were significantly attenuated by treatment with propofol ( $\mathrm{P}$ $<0.05$; Fig. $1 \mathrm{~B}$ and $\mathrm{C}$ ).

Propofol protects hippocampal neurons in IBI via the upregulation of GLT-1. To determine whether propofol protects hippocampal neurons in IBI via the upregulation of GLT-1, western blot analysis and RT-qPCR were performed. It was found that the protein and mRNA expression levels of GLT-1 were significantly decreased in the IBI group compared with the control group $(\mathrm{P}<0.05$; Fig. $2 \mathrm{~A}$ and $\mathrm{B})$. The decrease in GLT-1 expression caused by IBI was significantly attenuated by treatment with propofol (IBI $+\mathrm{P}$ group) compared with the IBI group ( $\mathrm{P}<0.05$; Fig. $2 \mathrm{~A}$ and $\mathrm{B})$. GLT-1 silencing by siRNA and the overexpression of GLT-1 was established by transfection with GLT-1-pIRES vector or siRNA against GLT-1. The efficiency of GLT-1 overexpression and silencing was detected by western blot analysis and RT-qPCR. The results revealed that the protein expression of GLT-1 correlated with its mRNA expression (Fig. 2C and D). The protein and mRNA expression of GLT-1 did not differ significantly among the control, scramble and empty vector groups (Fig. 2C and D). The expression of GLT-1 was significantly decreased in the si-GLT-1 group compared with the control group $(\mathrm{P}<0.05)$ and it was increased in the GLT-1 group compared with the control group $(\mathrm{P}<0.05$; Fig. $2 \mathrm{C}$ and $\mathrm{D})$. Cell apoptosis and viability were examined in the hippocampal neurons following GLT-1 silencing under hypoxic conditions. As shown in the dual-parameter fluorescent dot plots and bar graph, cell apoptosis was not affected by transfection with control siRNA (Scramble + IBI group or the Scramble + IBI + P group compared with the IBI and IBI $+\mathrm{P}$ groups, respectively). The knockdown of GLT-1 markedly increased the apoptotic rate (si-GLT-1 + IBI and si-GLT-1 + IBI + P groups compared with the IBI and $\mathrm{IBI}+\mathrm{P}$ groups, respectively) $(\mathrm{P}<0.05$; Fig. $2 \mathrm{E}$ and $\mathrm{F})$. Propofol did not attenuate the increase in the apoptotic rate when GLT-1 was knocked down (Fig. 2E and F). In the cell viability assay, treatment with propofol yielded similar results to those of the apoptosis assay (Fig. 2G). The si-GLT-1 + IBI and si-GLT-1 + IBI + P groups exhibited markedly reduced cell survival rates compared with the IBI and IBI + P groups, respectively $(\mathrm{P}<0.05$; Fig. $2 \mathrm{G})$. The effect of GLT-1 knockdown was slightly reversed by propofol (Fig. 2G). 

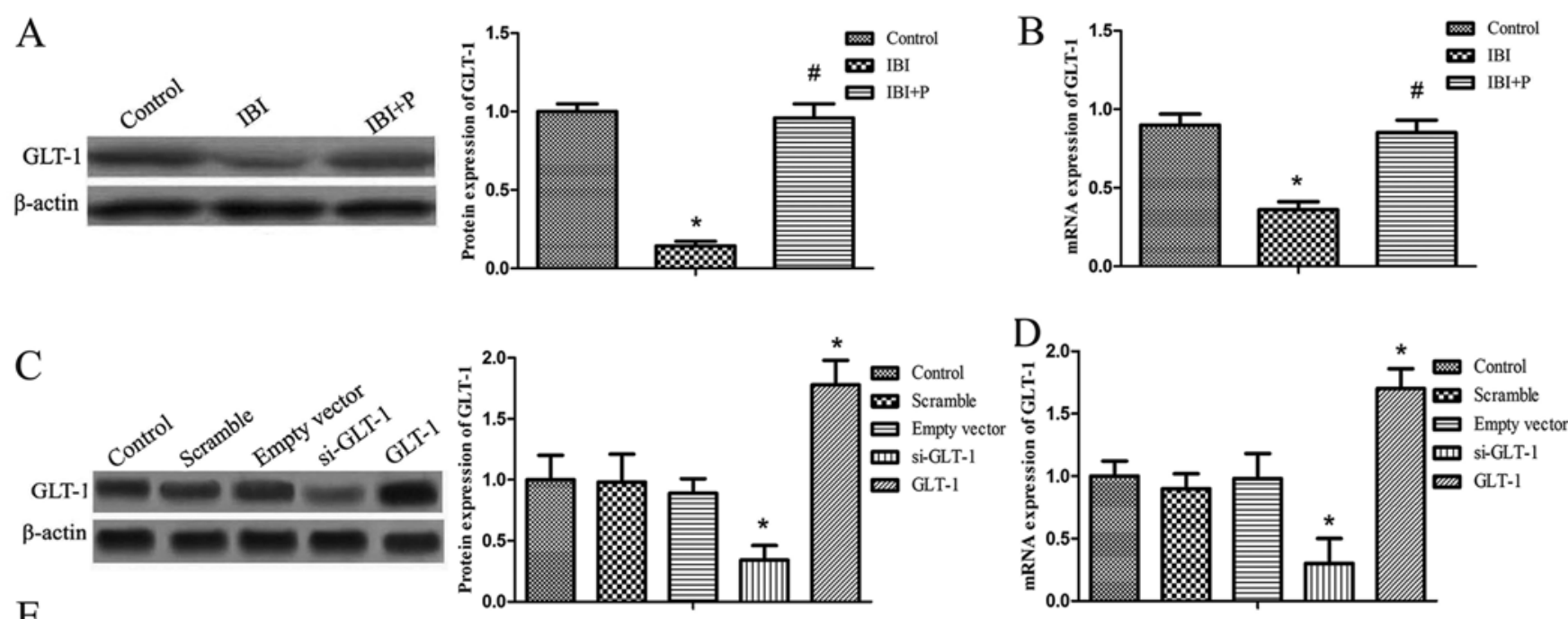

E

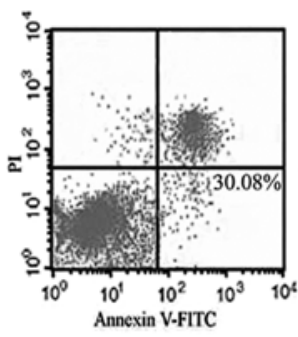

IBI

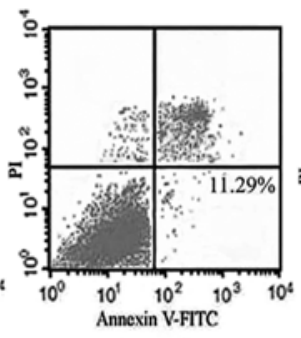

IBI+P

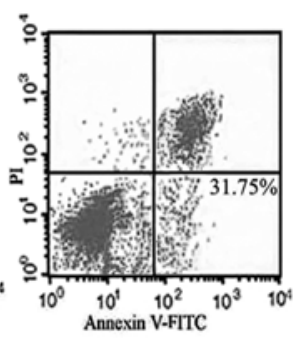

Scramble+IBI

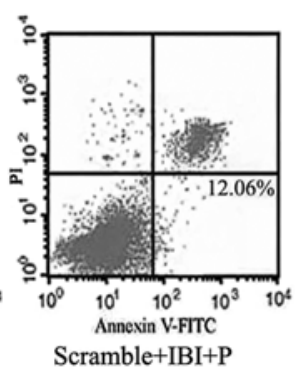

$\mathrm{G}$
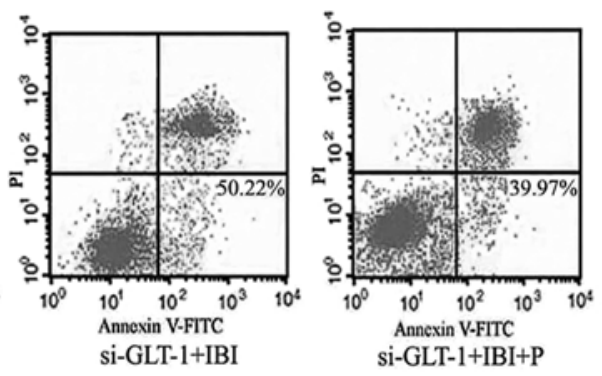

F

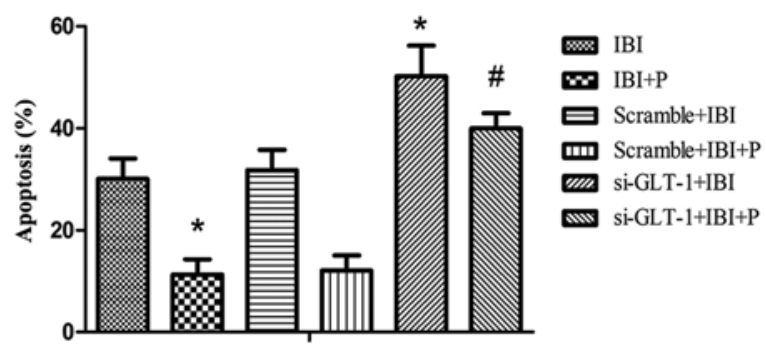

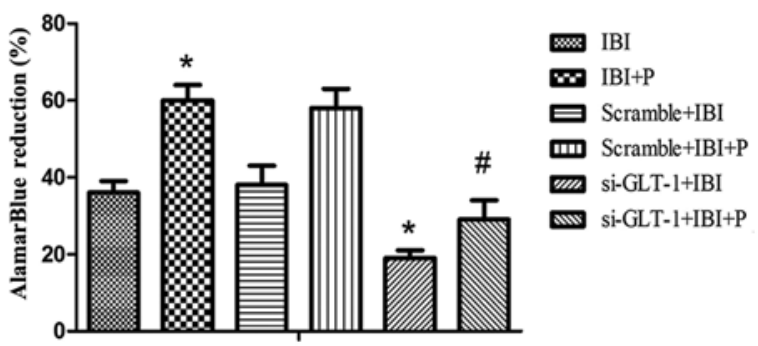

Figure 2. Propofol reverses the effect of glial glutamate transporter-1 (GLT-1) knockdown on hippocampal neuron cells in ischemic brain injury (IBI). All groups were treated on the basis of IBI except for the control group and the following groups. The scramble group, the empty vector group, the si-GLT-1 group and the GLT-1 group were treated under normoxic conditions. (A) The protein expression of GLT-1 in hippocampal neurons with propofol treatment was determined by western blot analysis. $\beta$-actin was utilized as an internal control. (B) The mRNA expression of GLT-1 in hippocampal neurons with propofol treatment was determined by RT-qPCR. GAPDH was utilized as an internal control. (C) The protein expression of GLT-1 following overexpression and silencing was determined by western blot analysis. $\beta$-actin was utilized as an internal control. (D) The mRNA expression of GLT-1 following overexpression and silencing was determined by RT-qPCR. GAPDH was utilized as an internal control. ${ }^{*} \mathrm{P}<0.05$ vs. control group; ${ }^{\#} \mathrm{P}<0.05$ vs. IBI group. (E) Cell apoptosis was detected using Annexin V-FITC/propidium iodide (PI) by flow cytometry in dual-parameter fluorescent dot plots. (F) Quantitative graphs of (E). (G) Cell viability was detected using AlamarBlue. " $\mathrm{P}<0.05$ vs. IBI group; ${ }^{\#} \mathrm{P}<0.05$ vs. IBI $+\mathrm{P}$ group.

JNK/Akt signaling pathway is involved in propofol-mediated neuroprotection. Our findings revealed that propofol protected the nerve cells against ischemic injury via the upregulation of the expression of GLT-1. To explore the potential signaling pathways through which propofol upregulated GLT-1 expression in our model of IBI in hippocampal neuronal cells, the expression of JNK, p-JNK, p38, p-p38, ERK1/2, p-ERK1/2, Akt and p-Akt was detected by western blot analysis (Fig. 3A). It was found that the expression of p38, ERK1/2, JNK and Akt exhibited no obvious change between the groups, whereas the phosphorylation levels of p38, ERK1/2, JNK and Akt exhibited marked differences in some groups (Fig. 3B-E). The levels of p-p38, p-ERK1/2 and p-JNK in the IBI + P group were signifi- cantly decreased when compared to the IBI group $(\mathrm{P}<0.05$; Fig. 3B-D), while the levels of p-Akt were significantly increased in the IBI + $\mathrm{P}$ group compared with IBI group $(\mathrm{P}<0.05$; Fig. 3E). Following the knockdown of GLT-1, no significant differences in the levels of p-p38 and p-ERK1/2 were observed between the si-GLT-1 + IBI and IBI groups, and between the si-GLT-1 + IBI + P and IBI + P groups (Fig. 3B and C). However, GLT-1 knockdown significantly increased the levels of p-JNK in the si-GLT-1 + IBI and si-GLT-1 + IBI + P groups compared with IBI and IBI $+\mathrm{P}$ groups, respectively $(\mathrm{P}<0.05$; Fig. 3D). GLT-1 knockdown led to a significant decrease in the levels of p-Akt in the si-GLT-1 + IBI and si-GLT-1 + IBI + P groups compared with the IBI and IBI + $\mathrm{P}$ groups, respec- 

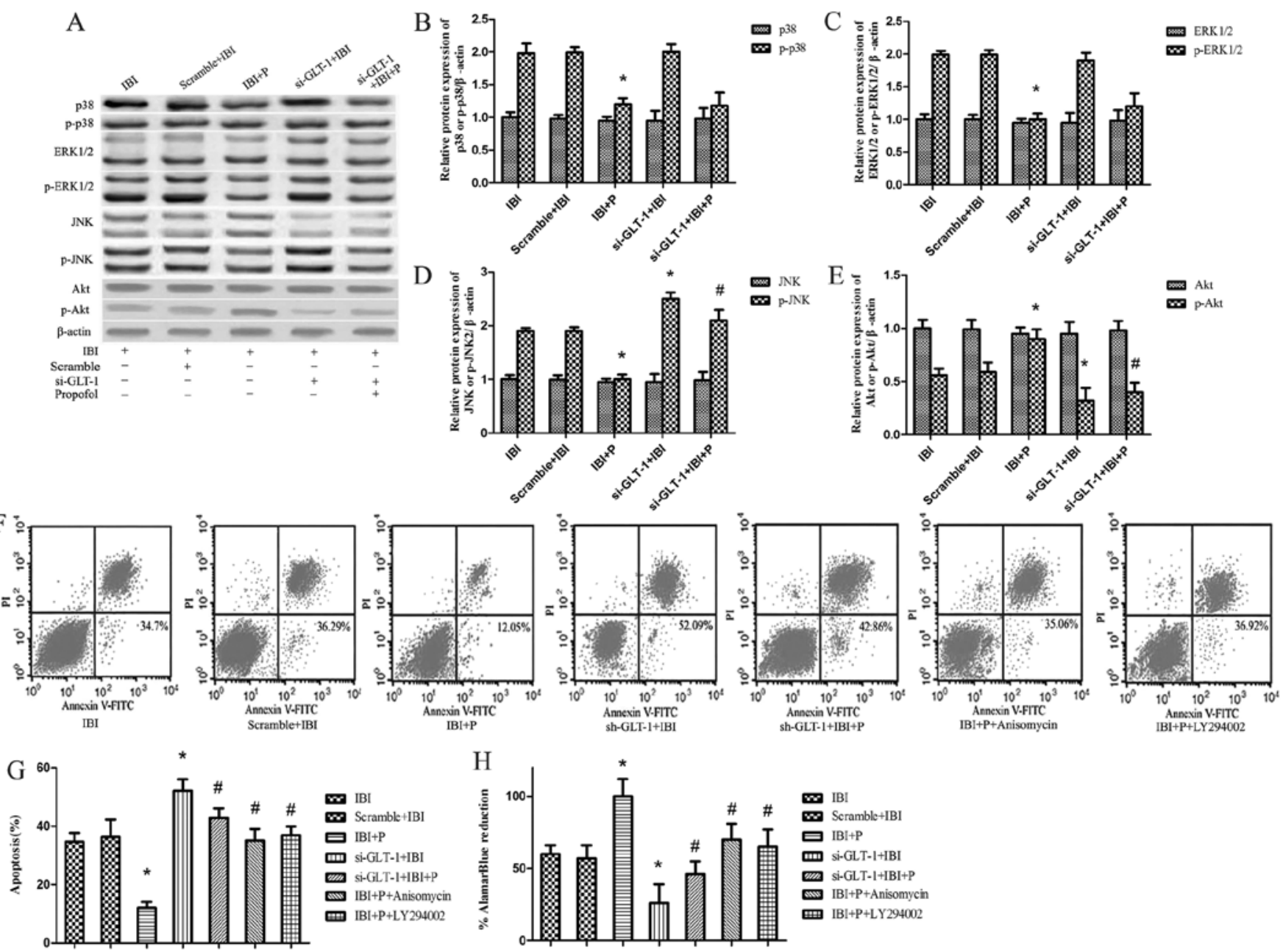

Figure 3. Signaling pathways of propofol-mediated neuroprotection under glial glutamate transporter-1 (GLT-1) knockdown conditions. Ischemic brain injury (IBI) + P+ anisomycin group cells were treated with propofol and the Jun N-terminal kinases (JNK) agonist, anisomycin, under hypoxic conditions. IBI + P + LY294002 group cells were treated with propofol and the Akt inhibitor, LY294002, under hypoxic conditions. (A) The protein expression of ERK, p-ERK, p38, p-p38, JNK, p-JNK, Akt and p-Akt was determined by western blot analysis with corresponding antibodies. $\beta$-actin was utilized as an internal control. (B-E) These data were calculated using ImageJ software. (F) Cell apoptosis was detected in the presence of anisomycin and LY294002 by Annexin V-FITC/propidium iodide (PI) in dual-parameter fluorescent dot plots. (G) Quantitative graphs of (F). (H) Cell viability was detected using AlamarBlue. ${ }^{*} \mathrm{P}<0.05$ vs. IBI group; ${ }^{\text {}} \mathrm{P}<0.05$ vs. IBI $+\mathrm{P}$ group.

tively $(\mathrm{P}<0.05$; Fig. 3E). No significant differences in the levels of $\mathrm{p}$-Akt were observed between the si-GLT-1 + IBI and si-GLT-1 + IBI + P groups (Fig. 3E).

In order to further investigate the functional role of JNK and Akt in propofol-mediated neuroprotection, we examined the effects of the JNK agonist, anisomycin, and the Akt inhibitor, LY294002, on cell viability and cell apoptosis. It was found that the changes in cell viability and cell apoptosis did not differ significantly between the IBI and Scramble + IBI groups (Fig. 3F-H). The increase in cell apoptosis and the decrease in cell viability were significantly attenuated by propofol in the IBI $+\mathrm{P}$ group compared with the IBI group $(\mathrm{P}<0.05$; Fig. $3 \mathrm{G}$ and $\mathrm{H})$. The decrease in cell apoptosis and the increase in cell viability caused by propofol were attenuated by anisomycin and LY294002, respectively $(\mathrm{P}<0.05$; Fig. $3 \mathrm{G}$ and $\mathrm{H})$.

NMDAR plays a key role in propofol-mediated neuroprotection. To explore the role of NMDAR in propofol-mediated neuroprotection, the protein and mRNA expression levels of the NMDAR subunits NR1, NR2A and NR2B were detected in each group by western blot analysis and RT-qPCR. It was found that the protein expression level of NMDAR correlated with its mRNA expression level. There was a slight difference in the expression of NR2A between the groups (Fig. 4A-C). The protein and mRNA expression were coincident between the IBI and Scramble+IBI groups (Fig. 4A-C). Propofol significantly attenuated the increase in NR1 and NR2B expression caused by IBI in the IBI $+\mathrm{P}$ group compared with the IBI group $(\mathrm{P}<0.05$; Fig. $4 \mathrm{~B}$ and $\mathrm{C})$. This attenuation caused by propofol was significantly reversed by GLT-1 knockdown (si-GLT-1 + IBI + P group compared with IBI + $\mathrm{P}$ group) $(\mathrm{P}<0.05$; Fig. $4 \mathrm{~B}$ and $\mathrm{C})$. GLT-1 knockdown led to a significant increase in the levels of NR1 and NR2B in the si-GLT-1 + IBI group compared with the IBI group $(\mathrm{P}<0.05$; Fig. 4A-C). Propofol only slightly attenuated the increase in the levels of NR1 and NR2B caused by GLT-1 knockdown (si-GLT-1 + IBI + P group compared with the si-GLT-1 + IBI group) (Fig. 4B and C). As regards the levels of NR2A, no significant differences were observed between the groups. 

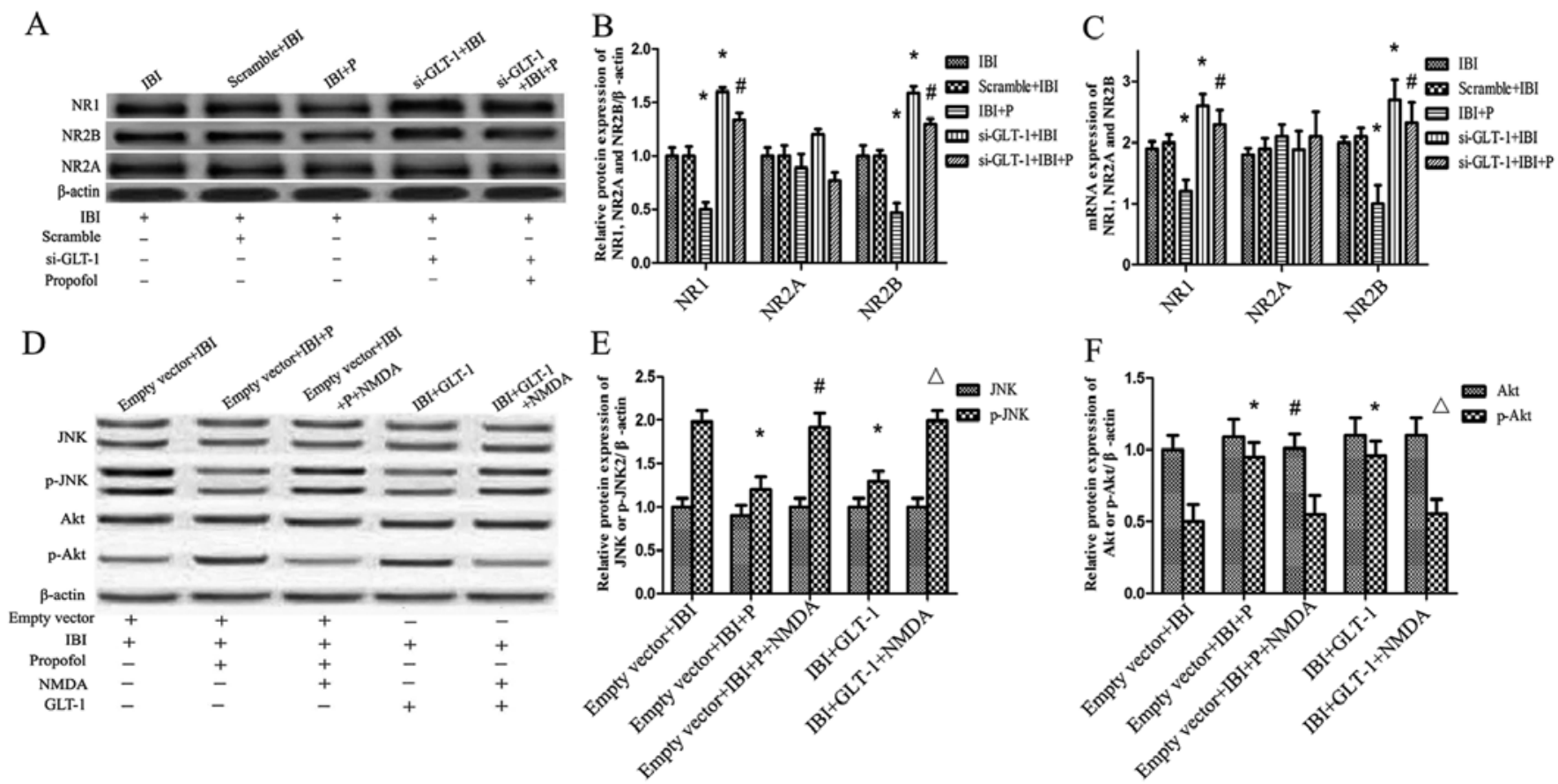

Figure 4. The protein and mRNA expression of N-methyl-D-aspartate (NMDA) receptor subunits NR1, NR2A, and NR2B and the expression of p-Jun N-terminal kinases (JNK) and p-Akt in the presence of NMDA. Ischemic brain injury (IBI) + glial glutamate transporter-1 (GLT-1) + NMDA group cells were treated with NMDA and pIRES-GLT-1 under hypoxic conditions. (A) The protein expression of NR1, NR2A, and NR2B was determined by western blot analysis with corresponding antibodies. $\beta$-actin was utilized as an internal control. (B) The data for protein expression were calculated using ImageJ software. (C) The mRNA expression of NR1, NR2A and NR2B was determined by RT-qPCR. GAPDH was utilized as an internal control. " $\mathrm{P}<0.05$ vs. IBI group; ${ }^{*} \mathrm{P}<0.05$ vs. IBI $+\mathrm{P}$ group. (D) The protein expression of JNK, p-JNK, Akt and p-Akt was determined by western blot analysis with corresponding antibodies. $\beta$-actin was utilized as an internal control. (E) The phosphorylation levels of JNK in the presence of NMDA were calculated using ImageJ software. (F) The phosphorylation levels of Akt in the presence of NMDA were calculated using ImageJ software. ${ }^{*} \mathrm{P}<0.05$ vs. empty vector $+\mathrm{IBI}$ group; ${ }^{"} \mathrm{P}<0.05$ vs. empty vector $+\mathrm{IBI}+\mathrm{P}$ group; ${ }^{\wedge} \mathrm{P}<0.05$ vs. IBI + GLT-1 group.

The activation of JNK and Akt was also detected in the presence of NMDA. As shown in Fig. 4D-F, the high level p-JNK and the low level p-Akt caused by IBI were significantly reversed by treatment with propofol (empty vector + IBI + P group compared with the empty vector + IBI group) $(\mathrm{P}<0.05)$. Treatment with NMDA significantly increased the levels of $\mathrm{p}-\mathrm{JNK}$ and decreased the levels of p-Akt (empty vector + IBI + P + NMDA group compared with the empty vector $+\mathrm{IBI}+\mathrm{P}$ group $)(\mathrm{P}<0.05$; Fig. $4 \mathrm{E}$ and $\mathrm{F})$. The effects of GLT-1 overexpression on the levels of p-JNK and p-Akt were similar to those observed with propofol treatment. GLT-1 overexpression led to a decrease in p-JNK levels and an increase in p-Akt levels (IBI + GLT-1 group compared with the IBI group) ( $\mathrm{P}<0.05$; Fig. 4E and F). NMDA significantly increased the levels of $\mathrm{p}-\mathrm{JNK}$ and decreased the levels of p-Akt (IBI + GLT-1 + NMDA group compared with IBI + GLT-1 group) $(\mathrm{P}<0.05$; Fig. 4E and F).

\section{Discussion}

IBI is a complex pathology that causes disability and dementia. Increasing evidence has indicated that IBI induces excessive extracellular glutamate release, which causes neurotoxicity and neuronal injury $(4,29)$. GLT-1 is one of the major glutamate transporters and GLT-1 can take up 90\% of glutamate, protecting against IBI $(30,31)$. Propofol is a commonly used clinical anesthetic. The neuroprotective effects of propofol have been previously demonstrated (32). However, it remains unclear as to whether propofol can regulate the expression of GLT-1 to protect neuronal cells in IBI.

In this study, the effects of propofol on neuronal cells in IBI were explored. The results revealed that hippocampal neuronal injury induced by hypoxia was attenuated by propofol. Consistent with our findings, the post-conditioning of rats with transient middle cerebral artery occlusion by propofol was shown to inhibit neuronal apoptosis in ischemia/reperfusion (33). Peroxynitrite-induced apoptosis in astroglial cells has also been shown to be attenuated by propofol treatment (34). An in vitro study demonstrated that propofol exerts a neuroprotective effect on hippocampal neurons against hypoxic damage (20). By contrast, a high dose propofol (200 $\mu \mathrm{M})$ has been shown to be cytotoxic in the neuroblastoma SH-SY5Y cell line (35).

The expression of GLT-1 was reduced in hippocampal neurons under hypoxic conditions. The decrease in GLT-1 expression caused by hypoxia was attenuated by propofol treatment in vitro. Similarly, propofol has been shown to attenuate the decrease in GLT-1 expression in depressed rats (21). The reduced cell viability and increased cell apoptosis caused by the downregulation of GLT-1 were markedly reversed by propofol treatment. It has also been shown that the knockdown of GLT-1 causes hippocampal excitotoxicity and exacerbates hippocampal neuronal cell damage and mortality, which are attenuated by propofol treatment $(21,36)$. However, when ischemia is elongated to a duration of $20 \mathrm{~min}$, GLT-1 leads to glutamate release and triggers neuronal death (8). 
Potential signaling pathways were explored by western blot analyssi. Previous studies have revealed that MAPK signaling pathways, particularly JNK, are involved in neuroprotection $(37,38)$. The functional relatedness of classical JNK-c-Jun signaling was restricted to the hypoxic-ischemic and excitotoxic sets of effects (37). The MAPK family includes JNK, the ERKs and p38 MAPK. Thus, the signaling pathway of p38, ERK1/2 and JNK was explored in the present study. In addition, Akt as a general signaling pathway in cell proliferation (39) and apoptosis (40) also was explored. The results of western blot analysis revealed that the p38, ERK1/2, JNK and Akt signaling pathways were involved in the propofol-mediated neuroprotection, whereas the increase in p-JNK and the decrease in p-Akt levels caused by GLT-1 knockdown were not attenuated by propofol. Similarly, the strong expression of the c-Jun gene and protein are known to precede or coincide with periods of intense cell death during embryonic development (41). JNK inhibition attenuated neuronal apoptosis in the developing rat brain after hypoxia-ischemia (42) and the activation of Akt decreased cell apoptosis (43). These findings suggested that the increase in the p-Akt level and the decrease in the p-JNK level were involved in neuroprotection (44). In this study, the JNK agonist, anisomycin, and the Akt, inhibitor LY294002, reversed the increase in p-Akt and the decrease in p-JNK levels caused by propofol treatment, suggesting that propofol protected hippocampal neurons by upregulating the expression of GLT-1 partly via the JNK/Akt signaling pathway.

A previous study showed that the excitotoxicity caused by the activation of NMDAR led to neuronal death following ischemia in some chronic neurodegenerative diseases (45). In our study, the excessive activation of NMDAR (NR1 and NR2B) caused by IBI was attenuated by propofol treatment. After GLT-1 knockdown, the effect of IBI on NMDAR was not reversed by propofol and GLT-1 knockdown led to an increase in NMDAR activation. Consistent with this study, it has been previously demonstrated that NMDAR, as the downstream molecule of GLT-1 (46), is possibly involved in propofol-mediated neuroprotection. In this study, NMDA reversed the decrease in p-JNK and the increase in p-Akt levels caused by propofol and GLT-1 overexpression. This result suggested that NMDA can induce JNK activation (15) and attenuate Akt activation (47) in IBI. It is implied that the NMDA receptor (NR1 and NR2B) could be the upstream molecule of JNK signaling and Akt signaling and, at least in part, may be involved in propofol-mediated neuroprotection.

In conclusion, the findings of our study suggest that propofol upregulates GLT-1 and inhibits NMDAR to protect hippocampal neuronal cells against IBI through the JNK/Akt signaling pathway. All experiments were performed in vitro, but the resulting details were not clear. We aim to perform in vivo experiments in future studies.

\section{References}

1. Bayat M, Azami Tameh A, Hossein Ghahremani M, Akbari M, Mehr SE, Khanavi M and Hassanzadeh G: Neuroprotective properties of Melissa officinalis after hypoxic-ischemic injury both in vitro and in vivo. Daru 20: 42, 2012.

2. Gong G, Yuan L, Cai L, Ran M, Zhang Y, Gong H, Dai X, Wu W and Dong H: Tetramethylpyrazine suppresses transient oxygenglucose deprivation-induced connexin 32 expression and cell apoptosis via the ERK1/2 and p38 MAPK pathway in cultured hippocampal neurons. PLoS One 9: e105944, 2014.
3. Chen H, Qu Y, Tang B, Xiong T and Mu D: Role of mammalian target of rapamycin in hypoxic or ischemic brain injury: Potential neuroprotection and limitations. Rev Neurosci 23: 279-287, 2012.

4. Choi DW and Rothman SM: The role of glutamate neurotoxicity in hypoxic-ischemic neuronal death. Annu Rev Neurosci 13: 171-182, 1990.

5. Gegelashvili G, Robinson MB, Trotti D and Rauen T: Regulation of glutamate transporters in health and disease. Prog Brain Res 132: 267-286, 2001.

6. Danbolt NC: Glutamate uptake. Prog Neurobiol 65: 1-105, 2001.

7. Ouyang YB, Xu L, Liu S and Giffard RG: Role of astrocytes in delayed neuronal death: GLT-1 and its novel regulation by MicroRNAs. Adv Neurobiol 11: 171-188, 2014.

8. Mitani A and Tanaka K: Functional changes of glial glutamate transporter GLT-1 during ischemia: An in vivo study in the hippocampal CA1 of normal mice and mutant mice lacking GLT-1. J Neurosci 23: 7176-7182, 2003.

9. Bruhn T, Levy LM, Nielsen M, Christensen T, Johansen FF and Diemer NH: Ischemia induced changes in expression of the astrocyte glutamate transporter GLT1 in hippocampus of the rat. Neurochem Int 37: 277-285, 2000.

10. Gong J, Gong S, Zhang M, Zhang L, Hu Y, Liu Y and Li W: Cerebral ischemic preconditioning reduces glutamate excitotoxicity by up-regulating the uptake activity of GLT-1 in rats. Amino Acids 46: 1537-1545, 2014.

11. Fang Q, Hu WW, Wang XF, Yang Y, Lou GD, Jin MM, Yan HJ, Zeng WZ, Shen Y, Zhang SH, et al: Histamine up-regulates astrocytic glutamate transporter 1 and protects neurons against ischemic injury. Neuropharmacology 77: 156-166, 2014.

12. Sun P, Zhang S, Li Y and Wang L: Harmine mediated neuroprotection via evaluation of glutamate transporter 1 in a rat model of global cerebral ischemia. Neurosci Lett 583: 32-36, 2014.

13. Hu YY, Xu J, Zhang M, Wang D, Li L and Li WB: Ceftriaxone modulates uptake activity of glial glutamate transporter-1 against global brain ischemia in rats. J Neurochem 132: 194-205, 2015.

14. Shen N, Mo LQ, Hu F, Chen PX, Guo RX and Feng JQ: A novel role of spinal astrocytic connexin 43: Mediating morphine antinociceptive tolerance by activation of NMDA receptors and inhibition of glutamate transporter-1 in rats. CNS Neurosci Ther 20: 728-736, 2014.

15. Nisticò R, Florenzano F, Mango D, Ferraina C, Grilli M, Di Prisco S, Nobili A, Saccucci S, D'Amelio M, Morbin M, et al: Presynaptic c-Jun N-terminal Kinase 2 regulates NMDA receptor-dependent glutamate release. Sci Rep 5: 9035, 2015.

16. Barone FC and Feuerstein GZ: Inflammatory mediators and stroke: New opportunities for novel therapeutics. J Cereb Blood Flow Metab 19: 819-834, 1999.

17. Furuya $T$, Pan $Z$ and Kashiwagi K: Role of retinal glial cell glutamate transporters in retinal ganglion cell survival following stimulation of NMDA receptor. Curr Eye Res 37: 170-178, 2012.

18. Harman F, Hasturk AE, Yaman M, Arca T, Kilinc K, Sargon MF and Kaptanoglu E: Neuroprotective effects of propofol, thiopental, etomidate, and midazolam in fetal rat brain in ischemia-reperfusion model. Childs Nerv Syst 28: 1055-1062, 2012.

19. Zhou R, Yang Z, Tang X, Tan Y, Wu X and Liu F: Propofol protects against focal cerebral ischemia via inhibition of microglia-mediated proinflammatory cytokines in a rat model of experimental stroke. PLoS One 8: e82729, 2013.

20. Zhang DX, Ding HZ, Jiang S, Zeng YM and Tang QF: An in vitro study of the neuroprotective effect of propofol on hypoxic hippocampal slice. Brain Inj 28: 1758-1765, 2014.

21. Zhu X, Hao X, Luo J, Min S, Xie F and Zhang F: Propofol inhibits inflammatory cytokine-mediated glutamate uptake dysfunction to alleviate learning/memory impairment in depressed rats undergoing electroconvulsive shock. Brain Res 1595: 101-109, 2015.

22. Hama-Tomioka K, Kinoshita $H$, Nakahata $K$, Kondo $T$, Azma T, Kawahito S, Hatakeyama N and Matsuda N: Roles of neuronal nitric oxide synthase, oxidative stress, and propofol in N-methyl-D-aspartate-induced dilatation of cerebral arterioles. Br J Anaesth 108: 21-29, 2012.

23. Kingston S, Mao L, Yang L, Arora A, Fibuch EE and Wang JQ: Propofol inhibits phosphorylation of N-methyl-D-aspartate receptor NR1 subunits in neurons. Anesthesiology 104: 763-769, 2006.

24. Brewer GJ: Serum-free B27/neurobasal medium supports differentiated growth of neurons from the striatum, substantia nigra, septum, cerebral cortex, cerebellum, and dentate gyrus. J Neurosci Res 42: 674-683, 1995.

25. Junghans U and Kappler J: Rat neocortex. In: The neuron in tissue culture, IBRO Handbook series: Methods in the neurosciences. Haynes LW (ed). John Wiley and Sons. Vol 18. Chichester, pp545-553, 1999. 
26. Cui D, Wang L, Qi A, Zhou Q, Zhang X and Jiang W: Propofol prevents autophagic cell death following oxygen and glucose deprivation in PC12 cells and cerebral ischemia-reperfusion injury in rats. PLoS One 7: e35324, 2012.

27. Livak KJ and Schmittgen TD: Analysis of relative gene expression data using real-time quantitative PCR and the 2(-Delta Delta C(T)) method. Methods 25: 402-408, 2001.

28. Suchanek W and Yoshimura M: Processing and properties of hydroxyapatite-based biomaterials for use as hard tissue replacement implants. J Mater Res 13: 94-117, 1998.

29. Hinzman JM, Thomas TC, Quintero JE, Gerhardt GA and Lifshitz J: Disruptions in the regulation of extracellular glutamate by neurons and glia in the rat striatum two days after diffuse brain injury. J Neurotrauma 29: 1197-1208, 2012.

30. Tanaka K, Watase K, Manabe T, Yamada K, Watanabe M, Takahashi K, Iwama H, Nishikawa T, Ichihara N, Kikuchi T, et al: Epilepsy and exacerbation of brain injury in mice lacking the glutamate transporter GLT-1. Science 276: 1699-1702, 1997.

31. Kim K, Lee SG, Kegelman TP, Su ZZ, Das SK, Dash R, Dasgupta S, Barral PM, Hedvat M, Diaz P, et al: Role of excitatory amino acid transporter-2 (EAAT2) and glutamate in neurodegeneration: Opportunities for developing novel therapeutics. J Cell Physiol 226: 2484-2493, 2011.

32. Fan W, Zhu X, Wu L, Wu Z, Li D, Huang F and He H: Propofol: An anesthetic possessing neuroprotective effects. Eur Rev Med Pharmacol Sci 19: 1520-1529, 2015.

33. Wang HY, Wang GL, Yu YH and Wang Y: The role of phosphoinositide-3-kinase/Akt pathway in propofol-induced postconditioning against focal cerebral ischemia-reperfusion injury in rats. Brain Res 1297: 177-184, 2009.

34. Acquaviva R, Campisi A, Raciti G, Avola R, Barcellona ML, Vanella L and Li Volti G: Propofol inhibits caspase-3 in astroglia cells: Role of heme oxygenase-1. Curr Neurovasc Res 2: 141-148, 2005.

35. Wu GJ, Chen WF, Hung HC, Jean YH, Sung CS, Chakraborty C, Lee HP, Chen NF and Wen ZH: Effects of propofol on proliferation and anti-apoptosis of neuroblastoma SH-SY5Y cell line: New insights into neuroprotection. Brain Res 1384: 42-50, 2011.

36. Rao VL, Dogan A, Todd KG, Bowen KK, Kim BT, Rothstein JD and Dempsey RJ: Antisense knockdown of the glial glutamate transporter GLT-1, but not the neuronal glutamate transporter EAAC1, exacerbates transient focal cerebral ischemia-induced neuronal damage in rat brain. J Neurosci 21: 1876-1883, 2001.
37. Raivich G: c-Jun expression, activation and function in neural cell death, inflammation and repair. J Neurochem 107: 898-906, 2008.

38. Zhu Z, Li R, Stricker R and Reiser G: Extracellular $\alpha$-crystallin protects astrocytes from cell death through activation of MAPK, PI3K/Akt signaling pathway and blockade of ROS release from mitochondria. Brain Res 1620: 17-28, 2015.

39. Liu K, Zhang Q, Lan H, Wang L, Mou P, Shao W, Liu D, Yang W, Lin Z, Lin Q, et al: GCN5 Potentiates Glioma Proliferation and Invasion via STAT3 and AKT Signaling Pathways. Int J Mol Sci 16: 21897-21910, 2015.

40. Gu Q, Zhai L, Feng X, Chen J, Miao Z, Ren L, Qian X, Yu J, Li Y, $\mathrm{Xu} \mathrm{X}$ and Liu CF: Apelin-36, a potent peptide, protects against ischemic brain injury by activating the PI3K/Akt pathway. Neurochem Int 63: 535-540, 2013.

41. Sun W, Gould TW, Newbern J, Milligan C, Choi SY, Kim H and Oppenheim RW: Phosphorylation of c-Jun in avian and mammalian motoneurons in vivo during programmed cell death: An early reversible event in the apoptotic cascade. J Neurosci 25: 5595-5603, 2005

42. Li D, Li X, Wu J, Li J, Zhang L, Xiong T, Tang J, Qu Y and Mu D Involvement of the JNK/FOXO3a/Bim pathway in neuronal apoptosis after hypoxic-ischemic brain damage in neonatal rats. PLoS One 10: e0132998, 2015.

43. Tovilovic G,Zogovic N, Soskic V, Schrattenholz A, Kostic-Rajacic S, Misirkic-Marjanovic M, Janjetovic K, Vucicevic L, Arsikin K, Harhaji-Trajkovic L and Trajkovic V: Arylpiperazine-mediated activation of Akt protects SH-SY5Y neuroblastoma cells from 6-hydroxydopamine-induced apoptotic and autophagic death Neuropharmacology 72: 224-235, 2013.

44. Xu L, Li Y, Fu Q and Ma S: Perillaldehyde attenuates cerebral ischemia-reperfusion injury-triggered overexpression of inflammatory cytokines via modulating Akt/JNK pathway in the rat brain cortex. Biochem Biophys Res Commun 454: 65-70, 2014.

45. Zhou Q and Sheng M: NMDA receptors in nervous system diseases. Neuropharmacology 74: 69-75, 2013.

46. Joe N, Scott V and Brown CH: Glial regulation of extrasynaptic NMDA receptor-mediated excitation of supraoptic nucleus neurones during dehydration. J Neuroendocrinol 26: 35-42, 2014.

47. Dong C, Rovnaghi CR and Anand KJ: Ketamine affects the neurogenesis of rat fetal neural stem progenitor cells via the PI3K/Akt-p27 signaling pathway. Birth Defects Res B Dev Reprod Toxicol 101: 355-363, 2014. 